\title{
Tactual Recognition of Soft Objects from Deformation Cues
}

\author{
Liangliang Wang, Student Member, IEEE, Qiang Li, Member, IEEE, James Lam, Fellow IEEE, Zheng \\ Wang, Senior Member, IEEE
}

\begin{abstract}
Recognition of soft objects is a critical step to tailor policy for dexterous manipulation. Deformation property is one of the valuable tactile cues for inferring the identities of soft objects, especially those having similar appearance features. In this work, we implemented tactual grasping for discriminating soft objects from the response curves of indentation displacement in relation to grasping force. Unlike some existing methods defining certain local features from the force-displacement observations, we viewed the force-displacement curve as continuous function rather than discrete observations. We proposed to use functional data analysis for classifying the kind of force-displacement curve data. Functional principal component analysis (FPCA) was used to extract multivariate features from force-displacement curves. Different traditional machine learning models were trained for classifying the multivariate features and the best one was selected based on cross-validation. Case study demonstrated that our method could accurately distinguish eight different soft objects. The FPCA based features outperformed those manually defined features. We indicated that the force had crucial influence on the classification accuracy and proposed how to select the proper force for the classification task. Overall, this work provides practical guidelines on tactual recognition of soft objects.
\end{abstract}

Index Terms-Soft object recognition, tactual grasping, force-displacement curve, functional data analysis.

\section{INTRODUCTION}

$\mathrm{T}$ HE past decades have witnessed that the robotic applications were extended from manufacturing scenario to

Manuscript received: May 14, 2021; Revised August 9, 2021; Accepted September 27, 2021. This paper was recommended for publication by Editor Markus Vincze upon evaluation of the Associate Editor and Reviewers' comments. This work was supported in part by Science, Technology and Innovation Commission of Shenzhen Municipality under grant no. ZDSYS20200811143601004, NSFC Grant 51975268, Guangdong Provincial Key Laboratory of Human-Augmentation and Rehabilitation Robotics in Universities, Southern Marine Science and Engineering Guangdong Laboratory (Guangzhou), SUSTECH-AISONO Joint Lab, SUSTECH-Taizhou Innovation Fund, and SUSTECH Institute of Robotics Fund. (Corresponding author: Zheng Wang.)

L. Wang and J. Lam are with the Department of Mechanical Engineering, The University of Hong Kong, Hong Kong (email: wang1141101321@gmail.com, jlam@hku.hk).

Q. Li is with the Center for Cognitive Interaction Technology (CITEC), Bielefeld University, Bielefeld, Germany (email: qli@techfak.uni-bielefeld.de). $\mathrm{He}$ is supported by the "DEXMAN" project (Project no.: 410916101) funded by the Deutsche Forschungsgemeinschaft (DFG).

Z. Wang is with the Department of Mechanical and Energy Engineering, Southern University of Science and Technology, Shenzhen, China (email: wangz@sustech.edu.cn)

Digital Object Identifier (DOI): see top of this page. unstructured everyday life. One challenge for robots working in everyday life is that a large part of objects in such environments are soft, e.g., linear ropes, planar paper, cloths, and food [1]. Due to the objects' deformation, the complexity of interactions between the robot and soft objects increases [2]. In light of this, it is crucial to endow robotic systems with the capability of distinguishing soft objects so as to devise proper manipulation policy [3], [4]. For examples, agricultural robots are expected to be built with the ability of discriminating the fruits with different levels of ripeness and picking the ripe ones with proper contact force. Developing medical robots with capability of palpating the lumps in the subcutaneous tissue is helpful for diagnosis and treatment.

As illustrated in Fig. 1(a), deformation property is one of the valuable tactile cues that could be leveraged for recognizing different soft objects, especially those having similar appearance features (e.g., geometry and texture). Previous studies typically conducted tactual interactions (e.g., pressing, pinching) with an object to explore its deformation property under the exerted force. The interaction data such as indentation displacement, force, tactile images were collected [5-12]. By processing the interaction data, deformation property of the soft objects can be learned and classified. For example, some existing studies proposed to recognize different soft objects (e.g., silicon rubbers) by comparing their hardness, which was simply computed by the ratios of the force to the indentation displacement [13-18]. Some other approaches used regression model to fit the trace of force-displacement observations and they used the slope of the regression line as one-dimensional feature for classifying different objects [19-21]. Chitta et al. [22] also studied the force-position profiles and suggested a set of six generic tactile features for identifying deformable bottles and cans. While the above methods could be easily implemented, the features manually defined from the force-displacement curve data might lose crucial information and bias the classification result. Apart from the force-displacement information, advanced tactile images were also exploited [23], [24] for estimating the hardness of the soft objects. CNN was used to extract features from the raw tactile image sequence and then LSTM net was devised for predicting the hardness of silicone samples. Bhattacharjee et al. [25] employed LSTM net to handle the time-variant tactile sensing data to infer whether the object is hard or soft. 
(a) Tactual interaction

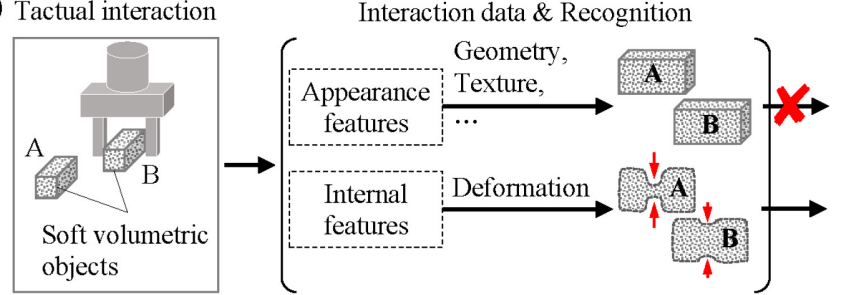

(b)

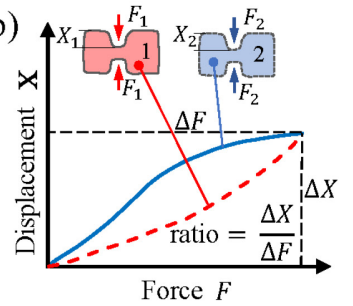

(c)

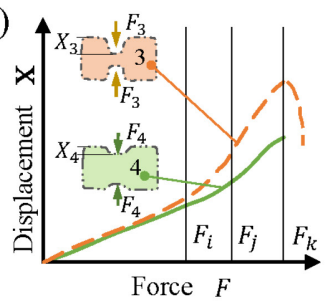

(d)
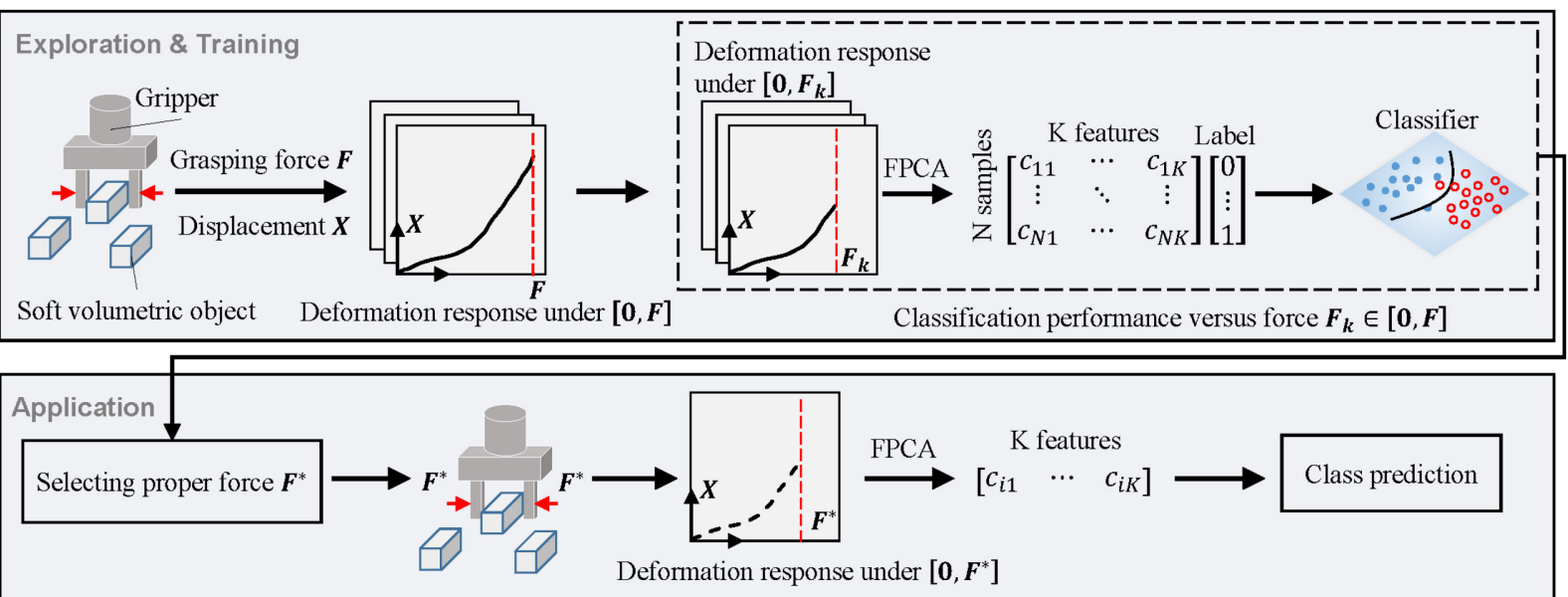

Fig. 1. Research motivation and tactual recognition scheme. (a) Deformation property is valuable for recognizing soft volumetric objects, especially those with similar appearance. (b) An example for illustrating the drawbacks of features that manually defined: ratio feature manually defined from force-displacement curve is ineffective for distinguishing the two objects. (c) An example for illustrating the effect of the force: small force results in poor discriminability while large force will cause unnecessary object deformation and even damage the object. (d) Scheme for tactual recognition of soft objects from deformation cues. Response curves of the indentation displacement in relation to grasping force were collected during the pinch grasping. FPCA was applied on classifying the force-displacement curve data. By investigating the classification accuracy in relation to the force, we indicated the guidelines on how to select the proper force to deform objects for classification purpose in practical applications.

In this work, we aim to recognize soft volumetric objects by learning their deformation property under the exerted force. Specifically, we explored the deformation properties of soft objects by pinching them using a two-finger proprioceptive gripper, which was prototyped in our previous studies [29], [30]. Response curves of the indentation displacement in relation to grasping force were collected for characterizing the deformation property of the soft objects. The focus of this work was providing a tool to process this type of force-displacement curve data for classifying different soft objects. We mainly tackled the following two issues:

(1) It is still an open question what features should be selected from raw force-displacement curve data for recognizing different soft objects. The prior works usually defined certain general characteristics or local features (e.g., ratio, slope, or $6 \mathrm{D}$ feature) from the force-displacement curve data for classification [12-22]. It is apt to lose crucial characteristics and bias the classification result. For instance, as illustrated in Fig. 1(b), the two objects have similar ratios of the force to the indentation displacement. Feature manually defined like ratio here has difficulty in discriminating them. But apparently, they are two types of soft objects with different deformation processes.

(2) Tactual interaction usually comes with object deformation, while few studies discussed how much force would be sufficient to deform the object for classification purpose. Fig. 1(c) illustrates this concern: insufficient deformation (when grasping force is less than $F_{i}$ ) may result in poor discriminability; too large grasping force, however, will cause unnecessary object deformation and even damage the object (when grasping force is larger than $F_{k}$ ).

Fig. 1(d) illustrates the proposed scheme for tactual recognition of soft objects. Our main contributions are summarized as following:

- We looked into the whole deformation process of the soft objects and treated the force-displacement curve data as continuous function rather than discrete observations. Under this perspective, we used functional data analysis for classifying the force-displacement curve data. Instead of manually defining certain local features from the force-displacement curves, we employed FPCA to generate a set of orthogonal basis functions for providing optimal approximations of the force-deformation curves. The coefficients of the basis functions explain the deformation behaviors of different objects and formed the multivariate features. The performance of classifying these features using different machine learning models were evaluated. As far as we know, in robotic tactual recognition field, this is the first trial to classify the force-displacement curve data under functional data analysis framework.

- The relationship between the classification accuracy and the amount of grasping force was investigated. In the case study, we indicated practical guidelines on how to select proper force to deform the objects and gain sufficient discriminable deformation features for classification purpose. 


\section{MODELS AND METHODS}

\section{A. Orthonormal Representation and Feature Extraction for Force-displacement Curves}

In this work, we looked into the whole deformation process of the soft objects and exploit the force-displacement curve data to discriminate objects. We consider the deformation observations as noisy sampled curves that realized from a smooth force-displacement function. Let $x_{i}(s)$ be the observation of the deformation curve sample $i$ at force $s$ :

$$
x_{i}(s)=X_{i}(s)+\varepsilon_{i}, i=1,2, \ldots, N ; s \in[0, F]
$$

where $X_{i}(s)$ is the uncontaminated curve, $[0, F]$ is the force interval, $N$ is the number of samples and $\varepsilon_{i}$ is white Gaussian noise. Using the Karhunen-Loève expansion, we can expand $X_{i}(s)$ by an infinite series of orthogonal basis functions $\left\{\phi_{k}(s), k \in \mathrm{N}\right\}$ :

$$
X_{i}(s)=\mu_{X}(s)+\sum_{k=1}^{\infty} C_{i k} \phi_{k}(s)
$$

where $\mu_{X}(s)$ is the mean of the curve samples and $C_{i k}=\int_{0}^{F}\left(X_{i}(s)-\mu_{X}(s)\right) \phi_{k}(s) d s, k \geq 1$. In FPCA [26-28], the process of mining the orthonormal system of eigenfunctions $\left\{\phi_{k}(s), k \in \mathrm{N}\right\}$ can be formulated as the following optimization problem:

$$
\max _{\int_{0}^{F} \phi(s)^{2} d s=1} \frac{1}{N-1} \sum_{i=1}^{N}\left[\int_{0}^{F}\left(X_{i}(s)-\mu_{X}(s)\right) \phi(s) d s\right]^{2}
$$

Consider the covariance function of $X(s)$

$$
\Gamma(v, s)=\operatorname{cov}(X(v), X(s))
$$

The maximization problem can be solved by finding a sequence of eigenvalue-eigenfunction pairs $\left\{\lambda_{k}, \phi_{k}(s)\right\}$ satisfying the eigenequation

$$
\int_{0}^{F} \Gamma(v, s) \phi_{k}(s) d s=\lambda_{k} \phi_{k}(s), 0 \leq v, s \leq F
$$

with $\lambda_{1} \geq \lambda_{2} \geq \ldots$ and eigenfunctions $\phi_{k}(s)$ are orthogonal, i.e., $\int_{0}^{F} \phi_{m}(s) \phi_{k}(s) d s=0$, if $m \neq k$. The coefficients $C_{i k}$ (called FPC score) is zero-mean uncorrelated random variable with variance $\lambda_{k}$ and can be considered as the projection of $X_{i}(s)$ on the eigenfunction $\phi_{k}(s)$. In practice, a finite number of eigenfunctions in Equation (5) are usually used to approximate $X_{i}(s)$. Denoting $X_{i}^{(K)}(s)$ as the approximation of $X_{i}(s)$ by truncating Equation (2) at the first $K$ terms, we have

$$
X_{i}^{(K)}(s) \approx \mu_{X}(s)+\sum_{k=1}^{K} C_{i k} \phi_{k}(s)
$$

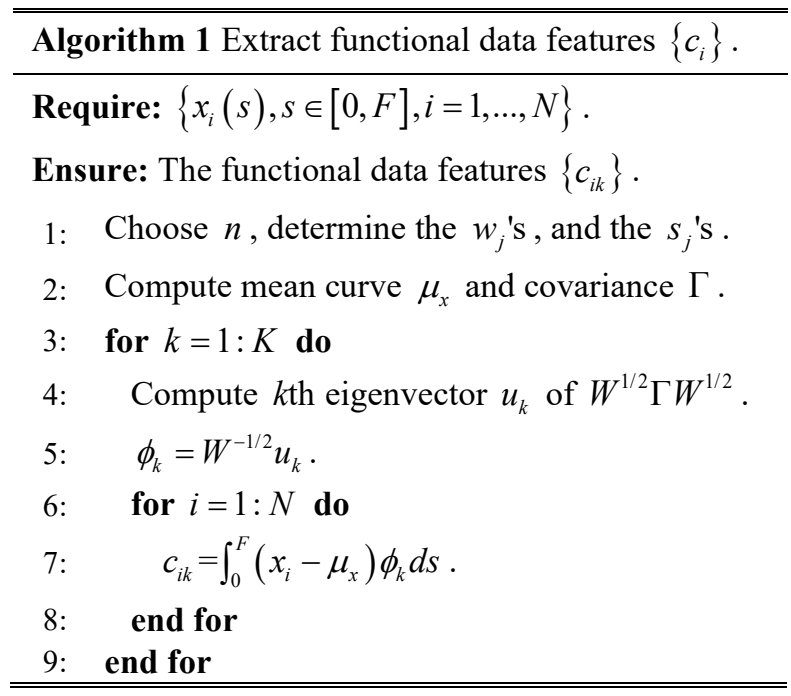

Here we use integral method to derive the eigenfunctions in Equation (6), which was proved to be computationally easy [26]. Suppose that the $N$ sample curves $x_{i}(s)$ are obtained with preliminary steps such as interpolation. The integral method is to discretize the observed curve $x_{i}(s)$ to $n$ equally spaced $s_{j}$ spanning the interval $[0, F]$, which yields $N \times n$ data matrix $X$. Let $h=F /(n-1)$ be the width of the equal interval, the integral equation in Equation (5) can be approximated as follows:

$$
\int_{0}^{F} \Gamma(v, s) \phi_{k}(s) d s \approx \sum_{\mathrm{j}} \Gamma\left(v, s_{j}\right) \phi_{k}\left(s_{j}\right) w_{j}
$$

where the weights $w_{j}$ are $h / 2, h, \ldots, h, h / 2$. Under the orthonormality constraints $\phi_{m}^{T} W \phi_{k}=\delta_{m k}\left(\delta_{m k}\right.$ is the Kronecker delta), the eigen analysis problem then can be written as equivalent matrix form:

$$
\Gamma W \phi_{k}=\lambda_{k} \phi_{k}
$$

where $\Gamma=\operatorname{cov}\left(\boldsymbol{X}\left(s_{i}\right), \boldsymbol{X}\left(s_{j}\right)\right), W=\operatorname{diag}\left(w_{1}, w_{2}, \ldots, w_{n}\right)$, and $\phi_{k}^{T}=\left(\phi_{k}\left(s_{1}\right), \phi_{k}\left(s_{2}\right), \ldots, \phi_{k}\left(s_{n}\right)\right)$.

Defining $u_{k}=W^{1 / 2} \phi_{k}$, we solve the symmetric eigenvalue problem of the form

$$
W^{1 / 2} \Gamma W^{1 / 2} u_{k}=\lambda_{k} u_{k}
$$

The vector $\phi_{k}$ can be recomputed via the inverse transformation $\phi_{k}=W^{-1 / 2} u_{k}$. Assume we select $K$ eigenfunctions $\phi_{1}, \phi_{2}, \ldots, \phi_{K}$ to form an orthonormal basis system, the corresponding FPC scores $\left(c_{i 1}, c_{i 2}, \ldots, c_{i K}\right)$ can be extracted as the functional features that representing $x_{i}(s), i=1,2, \ldots, N, s \in[0, F]$. Algorithm 1 presents the process of extracting the functional data features. 


\section{B. Classification Methods}

After the orthonormal representation, the functional force-displacement curve samples are converted to be the features in Euclidean space. In order to evaluate the effectiveness of the features, we choose five kinds of classification methods including support vector machine (SVM), K-nearest neighbor (KNN), Gaussian Naïve Bayes, multi-layer perceptron (MLP), and decision tree, for classifying the features. The classifiers were configured based on Scikit-learn (a machine learning library in Python [31]) and implemented on a common laptop with Intel Core i7-8550U CPU, 12GB RAM, and Intel UHD Graphics 620 in processor and Nvidia GeForce MX150.

SVM constructs a hyperplane to create the boundary between the data samples. Internally, the implementation is handled by using the library Libsvm. We trained the SVM classifiers with four different kernels, including linear kernel, radial basis function (RBF) kernel, polynomial kernel, and sigmoid kernel. The kernelized SVM was then constructed by selecting the kernel with the best classification performance in cross-validation.

KNN predicts the label of the new point from a predefined number of the training samples closest in distance. We used Euclidean distance and tunes the numbers of $\mathrm{K}$ by cross-validation.

Gaussian Naïve Bayes is based on applying Bayes' theorem with the assumption of independence among the features. In order to support the Gaussian Naïve Bayes for classification, the distribution of the features is assumed to be Gaussian.

MLP uses multi-layer network structure to learn a mapping from the input to output. To train the MLP classifier, one hidden layer was taken with ReLu activation function, learning rate is kept at 0.01 , and the parameters were optimized using the Adam algorithm.

Decision Tree creates a tree structure flowchart with a set of if-then-else decision rules for predicting the value of a target variable. We implemented the decision tree classifier with maximum depth of 5 .

\section{CASE STUdY}

In this section, we demonstrate the classification of eight different soft objects using the proposed method. Experimental setup for sampling the dataset was firstly introduced. Preliminary analysis of the dataset was carried out, following which we discussed the selection of classifier, the dimension of FPC components, and the grasping force. Finally, we compared the classification performance using our FPCA based features and the features manually defined in prior studies.

\begin{tabular}{|c|c|c|}
\hline Label & Object & Object section view (material \& internal structure) \\
\hline M & & - Medium density sponge \\
\hline H & & High density sponge \\
\hline MEM & & \begin{tabular}{|lll} 
& Medium density sponge \\
& Expanded polyethylene \\
\end{tabular} \\
\hline EPE & & - Expanded polyethylene \\
\hline So & & re A 0 silicon rubber \\
\hline S020 & & $\begin{array}{l}\text { - Shore A } 0 \text { silicon rubber } \\
\text { - Shore A } 20 \text { silicon rubber }\end{array}$ \\
\hline S10 & & - Shore A 10 silicon rubber \\
\hline $\mathrm{S} 10 \mathrm{H}$ & & Hollow inner cavity \\
\hline
\end{tabular}

Fig. 2. Eight classes of soft objects with similar appearance but different materials or internal structures. From the left to the right column: object labels, object sample image, and object section view for illustrating the materials and internal structures.

(a)

(c)
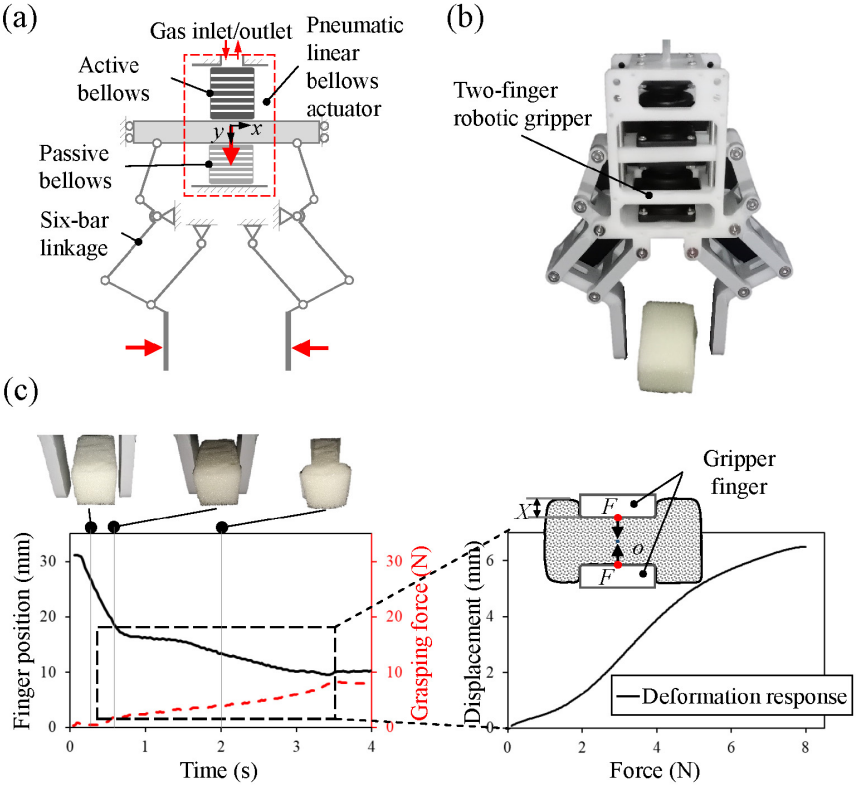

Fig. 3. Experimental exploration of objects' deformation properties using a two-finger robotic gripper. (a) Mechanism of the two-finger robotic gripper. (b) Prototype of the two-finger robotic gripper. (c) Pinch grasp exploration of a sponge sample. Deformation response of the sponge sample was delineated by plotting the indentation displacement of gripper finger as a function of the grasping force. 

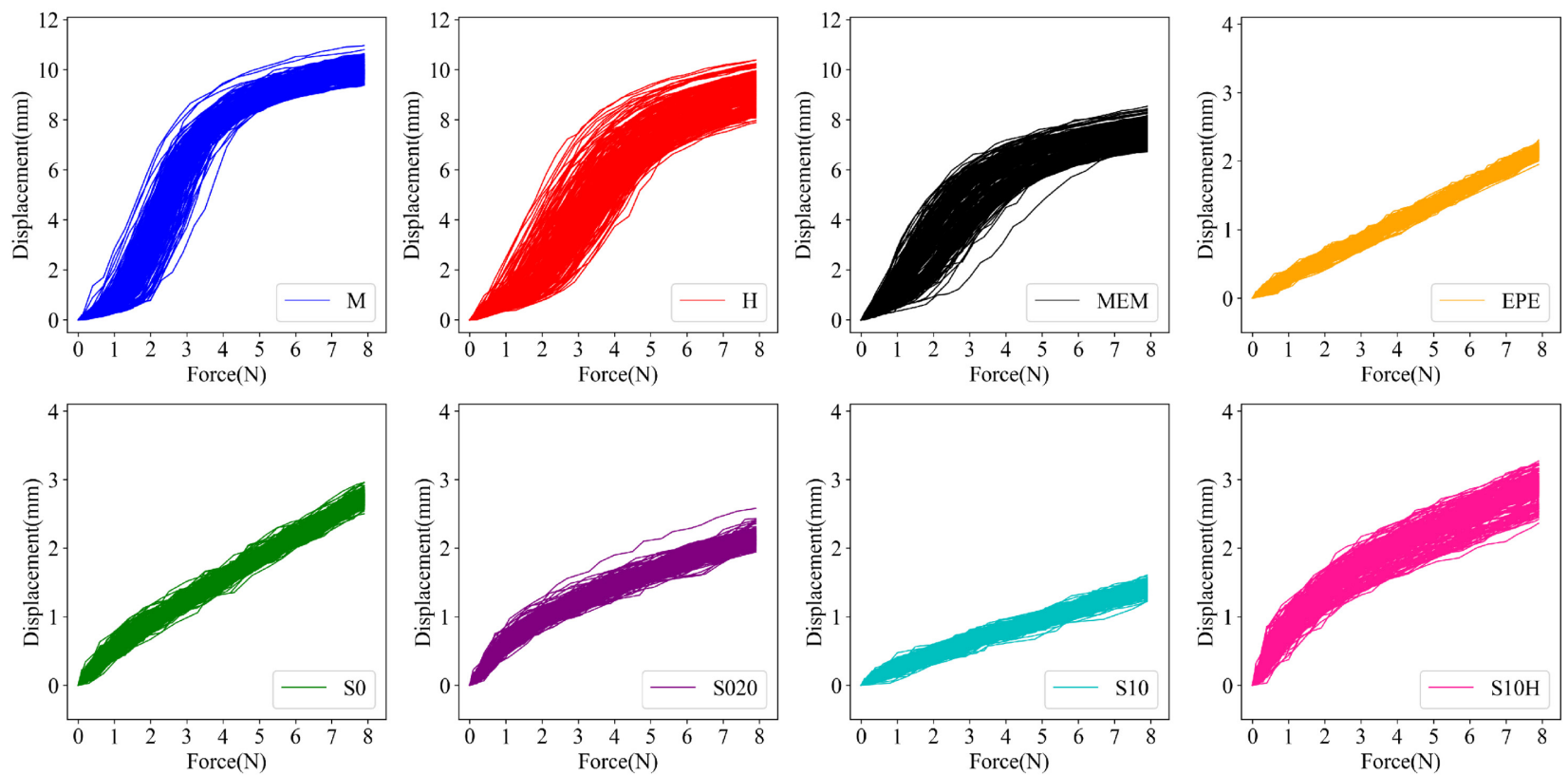

Fig. 4. Force-displacement curve data of the eight soft objects observed in grasping trials under controlled force in the range of $[0,8.0]$ (unit: Newton). For each class of object, 250 data curves were collected.

\section{A. Experimental Setup and Data Description}

We applied the proposed technique to classify eight soft objects with different physical properties merely by learning their deformation process. To avoid the influence of the appearance features, these objects were artificially made with similar shape, exterior size and color, based on which they could not be easily distinguished by computer vision techniques. But the interior of the eight soft objects were composed of different materials and structures, as illustrated by the object section view in Fig. 2. The eight objects were respectively labeled as \{"M", "H", "MEM", "EPE", "S0", "S020", "S10", "S10H"\}:

(1) object " $M$ " was made of medium density sponge;

(2) object " $\mathrm{H}$ " was made of high density sponge;

(3) object "MEM" was made of sandwich structure with medium density sponge on both sides and expanded polyethylene in the middle;

(4) object "EPE" was made of expanded polyethylene;

(5) object "S0" was made of Shore A 0 silicon rubber;

(6) object "S020" was constructed with the outer wall using Shore A 0 silicon rubber and the inner core using Shore A 20 silicon rubber;

(7) object "S10" was made of Shore A 10 silicon rubber;

(8) object "S10H" was constructed with the outer wall using Shore A 10 silicon rubber and a hollow inner cavity filled with air.

In this experiment, we used a two-finger robotic gripper to pinch the eight soft objects for characterizing their deformation properties. Fig. 3(a) and Fig. 3(b) respectively presents the mechanism and prototype of the gripper, which was constructed with six-bar linkages and pneumatically actuated by soft linear bellows chambers. By inflating and deflating the active bellows, the gripper finger was opened or closed for grasping objects. Two pressure sensors (range: $-100 \sim 300 \mathrm{kPa}$, CFSENSOR) were used for respectively monitoring the active and passive bellows' inner pressure. The pressure signals were collected and processed by a 32-bit dual-core MCU (EPS32, Espressif) for estimating the finger movement and grasping force. The estimation accuracies of force and displacement were strictly evaluated in our preliminary studies [29], [30]. In principle, the approach developed in this work can be used on other robot system with joint position measurement and calibrated tactile feedback.

Fig. 3(c) presents the experimental results of pinching a sponge. The sponge was symmetrically pinched while the indentation displacement of gripper finger and the corresponding grasping force were acquired by the MCU with sampling rate of $200 \mathrm{~Hz}$. After interpolation and plotting the observed indentation displacement of gripper finger in relation to the grasping force, a force-displacement curve was generated for delineating the deformation property of the sponge. The dataset for training and testing were acquired in the same manner. A total of 2000 grasp trials were carried out on the eight soft objects with 250 for each class. The grasping force was controlled in the range of $[0,8.0]$ (unit: Newton). To avoid biases, the pinching speed was randomized between the minimum of $2 \mathrm{~mm} / \mathrm{s}$ and the maximum of $10.5 \mathrm{~mm} / \mathrm{s}$. Fig. 4 shows the force-displacement curves of each class of object that collected in the grasping trials.

\section{B. Preliminary Analysis}

Preliminary analysis on the features extracted by FPCA was firstly carried out. It took about 8 milliseconds to compute the features. Fig. 5(a) reveals the cumulative proportion of variation explained by the first fifteen functional principal components (FPCs) and the proportion of variation that each FPC accounts for. The first three FPCs are plotted in Fig. 5(b). Fig 5(c) presents the pairwise scatterplots of the first three FPC scores of a training dataset that split in five-fold cross-validation. 

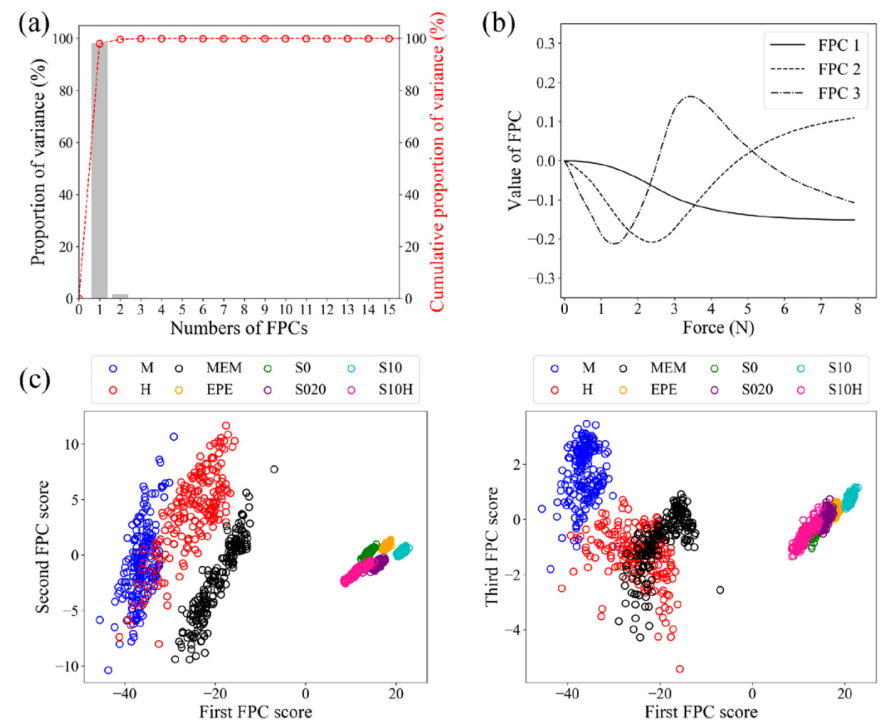

Fig. 5. (a) Cumulative proportion (curve plot) of variation explained by the first fifteen FPCs and the proportion (bar plot) of variation explained by each FPC. (b) The first three FPCs of the force-displacement curve data. (c) Pairwise scatterplots of the first three FPC scores of the training dataset.

According to Fig. 5(a), the first FPC explained $98.03 \%$ of the total variation, strongly dominating all other types of variation. The overall effect due to the first FPC increased with force increasing as shown in Fig. 5(b). It indicated that the object had high negative value of the first FPC score (e.g., the sponge material-based objects) would display larger deformation in the force range and those had high positive scores (e.g., the silicon rubber material-based objects) would display smaller deformation, as shown in Fig. 5(c). With the constraint of being orthogonal to the first FPC, the second FPC represented a mode of variation accounting for $1.66 \%$ of the total variation. As illustrated in Fig. 5(b), the second FPC consisted of negative contributions when force was less than about $5.0 \mathrm{~N}$, followed by a positive contribution. The object had high negative value of the second FPC score would display higher deformation rate up to $5.0 \mathrm{~N}$ and later gradually reverted to smaller deformation rate (e.g., the "S10H" object). The third FPC explained a very small variation with only $0.25 \%$. Objects with high positive values of the third FPC score would display higher deformation rate within the range of force less than around $2.5 \mathrm{~N}$.

\section{Selecting Dimension of FPCs and Classification Results}

Our preliminary analysis indicated that the first two FPCs could provide a good approximation of the raw curves since the percentage of total variance explained by them was over $99.5 \%$. But selecting dimension $K$ by reference to the proportion of variance explained is not geared toward minimizing classification error rates. Therefore, we used the five-fold cross-validation method to choose the dimension $K$.

The five classifiers were respectively trained with different numbers of FPCs. All the classifiers could be trained in 1 second with about $10 \mathrm{~ms}$ for Decision Tree, about $1000 \mathrm{~ms}$ for MLP, about $11 \mathrm{~ms}$ for Gaussian Naïve Bayes, about $1.5 \mathrm{~ms}$ for $\mathrm{KNN}$, and less than $80 \mathrm{~ms}$ for SVM (depended on the kernel function). In testing, the execution time of all the trained models for predicting the labels were less than $0.5 \mathrm{~ms}$.
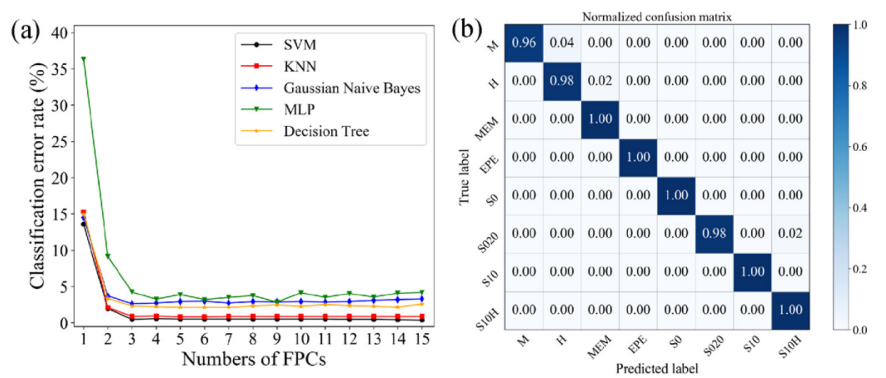

Fig. 6. (a) Relationship between the classification error rate of the five classifiers and the numbers of FPCs. Five-fold cross-validation was used. (b) Confusion matrix for evaluating classification accuracy of the best classifier (SVM with linear kernel) using the first three FPCs.

Fig. 6(a) gives the classification error rate of the five classifiers in relation to dimension $K$. The classification error rate decreased gradually with dimension $K$ increasing and little improvement was gained after $K=3$. Therefore, the first three FPCs were used for classification in this work. Cross-validation results indicated that SVM classifier (with linear kernel) outperformed the other four classifiers with the lowest mean classification error rate of $0.7 \%$ when $K=3$. Fig. 6(b) presents the classification results of the SVM classifier for a testing fold in five-fold cross-validation. According to the confusion matrix, all the "MEM", "EPE", "S0", "S10", "S10H" objects were correctly predicted. There were only $4 \%$ of " $\mathrm{M}$ ", $2 \%$ of " $\mathrm{H}$ ", and $2 \%$ of "S020" objects being misclassified.

\section{Selecting Sufficient Force to Gain Discriminable Deformation Profile}

We further investigated the relationship between the classification performance and the grasping force. The dilemma here was that insufficient force might result in poor discriminability while too large force might cause unnecessary object deformation and even damage the object. As showed in Fig. 4, the force-displacement curves of the eight objects overlapped at the early force step. With larger force, the force-displacement curves of different objects separated and the features became more and more distinct.

Using five-fold cross-validation, we plotted the classification error rate of the best classifier in relation to force and the numbers of FPCs in Fig. 7. With small force, little improvement could be made in the accuracy even more FPCs were used. The misclassification rate decreased monotonically with the force increasing and level off after $F=6.0$. When $F>6.0$, applying more force gained little improvement in classification accuracy. This indicated that the availability of deformation observations in the force range of $[0,6.0]$ (unit: Newton) contained adequate features to discriminate the eight classes of soft objects. For tactual recognition, force $6.0 \mathrm{~N}$ is sufficient to gain good classification results. 


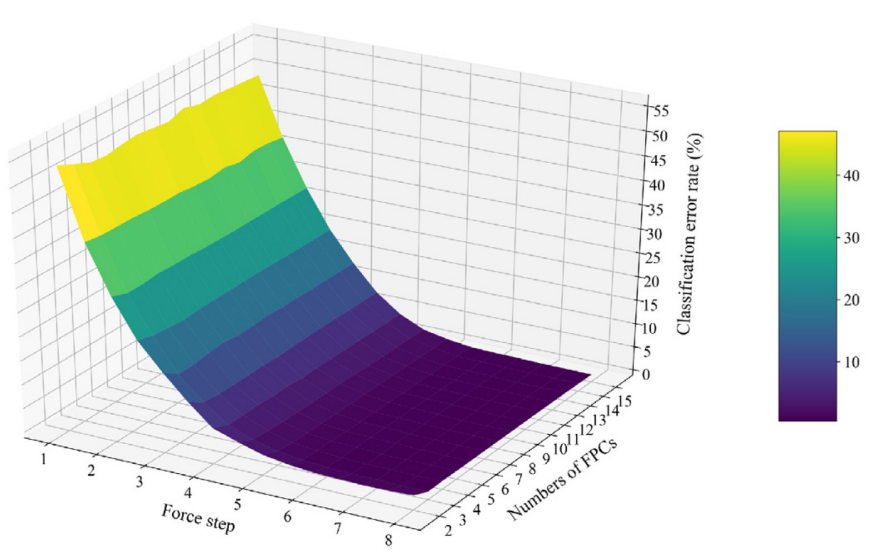

Fig. 7. Classification error rate of the best classifier in relation to force and the numbers of FPCs. Five-fold cross-validation was used.

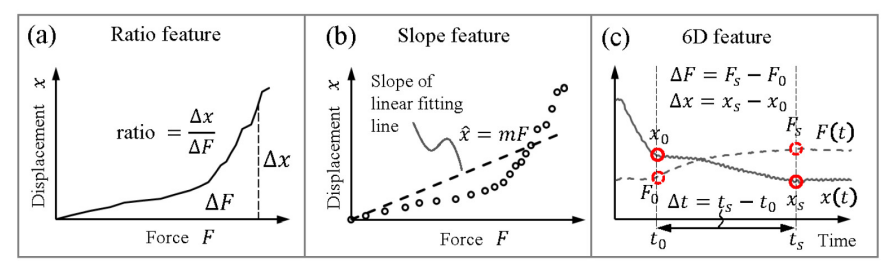

Fig. 8. The schematic of the features manually defined in prior works: (a) ratio feature; (b) slope feature; (c) 6D feature.

\section{E. Comparison with Features Manually Defined}

In addition to the FPCA based features, some previous studies manually defined features from the force-displacement curve data. Those features could be grouped into three categories - ratio feature [13-18], slope feature [19-21], and 6D feature [22], as illustrated in Fig. 8:

Ratio feature. This was 1-dimensional feature defined by ratio of the displacement change $\Delta x$ and force change $\Delta F$.

Slope feature. By fitting the force-displacement observations with linear regression model, the slope of the regression line was extracted as 1-dimensional feature.

6D feature. Chitta et al. [22] extracted 6-dimensional features from the sequential force $F(t)$ and position $x(t)$ observations, i.e., $a=\left(x_{0}, x_{s}, F_{s}, \Delta t, \Delta x / \Delta t, \Delta F / \Delta t\right)$.

For comparative purposes, we manually extracted these three categories of features from the force-displacement curve dataset. The five classifiers were trained and the best one was selected for classifying each type of feature respectively. Fig. 9(a) compares the classification performance of the best classifier of different features at every force step. Overall, the FPC feature outperformed the other three features. The ratio and $6 \mathrm{D}$ feature had similar performance, followed by the slope feature. To show the advantages of the FPC feature over the features manually defined, we go into the details of the classification results of the four features-based methods for a testing fold under the force range of $[0,6.0]$ (unit: Newton). As shown in Fig. 9(b), the force-displacement curves of the "M", "MEM", and "H" objects seriously overlapped each other over the whole force range. The "S0", "S020", and "S10H" objects also behaved with similar deformation response over the whole force range. This made it a challenging task to deliver the classification boundaries.
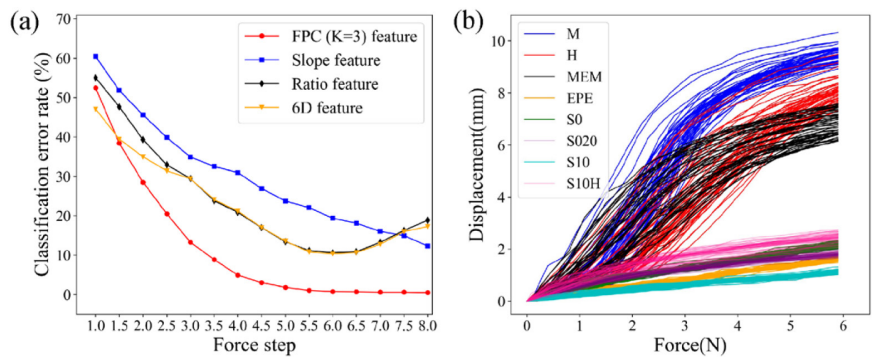

Fig. 9. (a) Classification error rate of the best classifiers of different features at every force step. Five-fold cross-validation was used. (b) A testing fold in five-fold cross-validation with force-displacement curves in the force range of $[0,6.0]$ (unit: Newton).
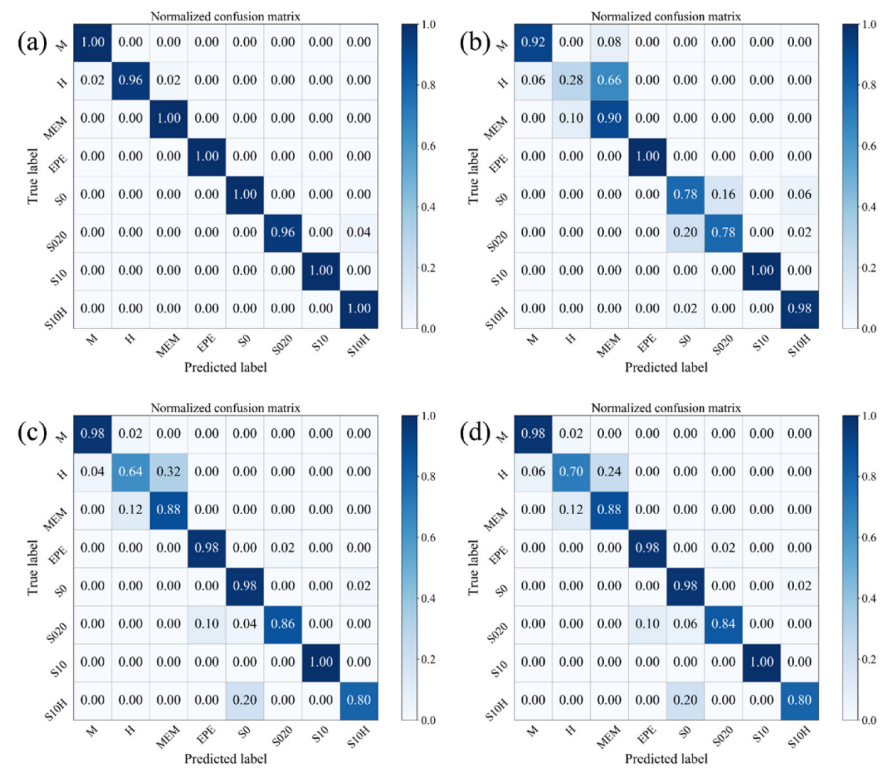

Fig. 10. Confusion matrix for evaluating the performance of the best classifiers of different features under the force range of $[0,6.0]$ (unit: Newton): (a) FPC feature-KNN; (b) slope feature-Gaussian Naïve Bayes; (c) ratio feature-Decision Tree; (d) 6D feature-SVM (linear kernel).

Fig. 10 presents the performance of the best classifiers of the four types of features under the force range of $[0,6.0]$. The FPC feature (best classifier is KNN) succeeded in solving this challenging task of distinguishing among different sponge material objects ("M", "MEM", and " $\mathrm{H}$ ") and among different silicon material objects ("S0", "S020", and "S10H") with only $4 \%$ of " $\mathrm{H}$ " and $4 \%$ of "S020" objects being misclassified. However, the three types of manually defined features showed poor discriminability. Based on the slope feature (best classifier is Gaussian Naïve Bayes), $72 \%$ of " $\mathrm{H}$ ", $12 \%$ of "MEM", $22 \%$ of "S0", and $22 \%$ of "S020" were misclassified. There were over $30 \%$ of "H", $12 \%$ of "MEM", around $15 \%$ of "S020", and $20 \%$ of "S10H" being incorrectly classified based on the ratio feature (best classifier is Decision Tree) or the 6D feature (best classifier is SVM with linear kernel).

The above results indicated us some guidelines: (1) sufficient deformation was required so as to gain evidently discriminable features for recognition purpose; (2) a proper feature extraction method helped capture the discriminant information in the deformation data. 


\section{CONCLUSION}

In this work, we provided a tactual scheme to enable robotic system to recognize soft objects based on their deformation properties. The deformation properties of the soft objects were explored by grasping and characterized by the response curves of the indentation displacement in relation to grasping force. We handled the force-displacement curve data as continuous function rather than discrete observations. FPCA was used to project the force-displacement curve data to multivariate features. To discriminate the multivariate features, different classifiers were built and the best one was selected based on cross-validation. Case study demonstrated that our method achieved high classification accuracy in discriminating eight different soft objects with similar geometry but different materials and internal structures. In comparison to those features manually defined in prior works, our FPCA based features could achieve better classification performance. Furthermore, we gave proposals on how to select proper grasping force to obtain good classification accuracy and efficiency.

In the future research, the object properties such as hysteresis and anisotropy observed in tactual exploration could also be leveraged for recognizing soft objects. In addition to the information of physical state, other tactile cues such as shape or texture may also be considered in the future. The current method may need to be improved for dealing with multiple dimensional input. Another interesting direction that could be examined is to use FPCA based method for estimating the magnitude of the softness of the soft objects.

\section{REFERENCES}

[1] J. Sanchez, J. A. Corrales, B. C. Bouzgarrou, and Y. Mezouar, "Robotic manipulation and sensing of deformable objects in domestic and industrial applications: a survey," Int. J. Robot. Res., vol. 37, no. 7, pp. 688-716, Jun. 2018.

[2] F. F. Khalil and P. Payeur, "Dexterous robotic manipulation of deformable objects with multi-sensory feedback - a review," in: Robot Manipulators Trends and Development, London: InTech, 2010.

[3] V. E. Arriola-Rios, P. Guler, F. Ficuciello, D. Kragic, and J. L. Wyatt, "Modeling of deformable objects for robotic manipulation: a tutorial and review," Front. Robot. AI, vol. 7, pp. 82, Sept. 2020.

[4] M. C. Gemici and A. Saxena, "Learning haptic representation for manipulating deformable food objects," in Proc. IEEE/RSJ Int. Conf. Intell. Robot. Syst., Chicago, IL, USA, 2014, pp. 638-645.

[5] S. Luo, J. Bimbo, R. Dahiya, and H. Liu, "Robotic tactile perception of object properties: a review," Mechatronics, vol. 48, pp. 54-67, Dec. 2017.

[6] Q. Li, O. Kroemer, Z. Su, F. F. Veiga, M. Kaboli, and H. J. Ritter, "A review of tactile information: perception and action through touch," IEEE Trans. Robot., vol. 36, no. 6, pp. 1619-1634, Dec. 2020

[7] B. Frank, R. Schmedding, C. Stachniss, M. Teschner, and W. Burgard, "Learning the elasticity parameters of deformable objects with a manipulation robot," in Proc. IEEE/RSJ Int. Conf. Intell. Robot. Syst., Taipei, Taiwan, 2010, pp. 1877-1883.

[8] P. Boonvisut and M. C. Cavuşoğlu, "Identification and active exploration of deformable object boundary constraints through robotic manipulation," Int. J. Robot. Res., vol. 33, no. 11, pp. 1446-1461, Sept. 2014.
[9] V. Chu, et al., "Robotic learning of haptic adjectives through physical interaction," Robot. Auton. Syst., vol. 63, pp. 279-292, Jan. 2015.

[10] P. Boonvisut and M. C. Cavuşoğlu, "Estimation of soft tissue mechanical parameters from robotic manipulation data," IEEE ASME Trans. Mechatron., vol. 18, no. 5, pp. 1602-1611, Oct. 2013.

[11] M. Ayyildiz, B. Guclu, M. Z. Yildiz, and C. Basdogan, "An optoelectromechanical tactile sensor for detection of breast lumps," IEEE Trans. Haptics, vol. 6, no. 2, pp. 145-155, Apr. 2013

[12] A. Drimus, G. Kootstra, A. Bilberg, and D. Kragic, "Design of a flexible tactile sensor for classification of rigid and deformable objects," Robot. Auton. Syst., vol. 62, no. 1, pp. 3-15, Jan. 2014.

[13] L. Huang, T. Kawamura, and H. Yamada, "Construction robot operation system with object's hardness recognition using force feedback and virtual reality," J. Robot. Mechatron., vol. 24, no. 6, pp. 958-966, Dec. 2012.

[14] Z. Su, J. A. Fishel, Y. Tomonori, and G. E. Loeb, "Use of tactile feedback to control exploratory movements to characterize object compliance," Front. Neurorobot., vol. 6, pp. 7, Jul. 2012.

[15] A. J. Spiers, M. V. Liarokapis, B. Calli, and A. M. Dollar, "Single-grasp object classification and feature extraction with simple robot hands and tactile sensors," IEEE Trans. Haptics, vol. 9, no. 2, pp. 207-220, Apr. 2016.

[16] L. Liu, Y. Zhang, G. Liu, and W. Xu, "Variable motion mapping to enhance stiffness discrimination and identification in robot hand teleoperation," Robot. Cim-Int. Manuf., vol. 51, pp. 202-208, Jun. 2018.

[17] D. L. Massimiliano, "Perceived compliance in a pinch," Vision Res., vol. 51, no. 8, pp. 961-967, Apr. 2011.

[18] W. M. Bergmann Tiest and A. M. L. Kappers, "Cues for haptic perception of compliance," IEEE Trans. Haptics, vol. 2, no. 4, pp. 189-199, Oct. 2009.

[19] M. Wouter and T. Bergmann. "Tactual perception of material properties," Vision Res., vol. 50, no. 24, pp. 2775-2782, Dec. 2010.

[20] L. Kaim and K. Drewing, "Exploratory strategies in haptic softness discrimination are tuned to achieve high levels of task performance," IEEE Trans. Haptics, vol. 4, no. 4, pp. 242-252, Oct. 2011.

[21] M. A. Srinivasan and R. H. LaMotte, "Tactual discrimination of softness," J. Neurophysiol., vol. 73, no. 1, pp. 88-101, Jan. 1995.

[22] S. Chitta, J. Sturm, M. Piccoli, and W. Burgard, "Tactile sensing for mobile manipulation," IEEE Trans. Robot., vol. 27, no. 3, pp. 558-568, Jun. 2011

[23] W. Yuan, C. Zhu, A. Owens, M. A. Srinivasan, and E. H. Adelson, "Shape-independent hardness estimation using deep learning and a GelSight tactile sensor," in Proc. IEEE Int. Conf. Robot. Autom., Singapore, Singapore, 2017, pp. 951-958.

[24] W. Yuan, M. A. Srinivasan, and E. H. Adelson, "Estimating object hardness with a GelSight touch sensor," in Proc. IEEE/RSJ Int. Conf. Intell. Robot. Syst., Daejeon, South Korea, 2016, pp. 208-215.

[25] T. Bhattacharjee, J. M. Rehg, and C. C. Kemp, "Inferring object properties with a tactile-sensing array given varying joint stiffness and velocity," Int. J. Humanoid Robot., vol. 15, no. 1, pp. 1750024, Feb. 2018.

[26] J. O. Ramsay and B. W. Silverman, "Applied functional data analysis: methods and case studies," New York, NY, USA: Springer, 2002.

[27] F. Ferraty and P. Vieu, "Nonparametric functional data analysis: theory and practice," New York, NY, USA: Springer, 2006.

[28] H. Li, G. Xiao, T. Xia, Y. Y. Tang, and L. Li, "Hyperspectral image classification using functional data analysis," IEEE Trans. Cybernetics, vol. 44, no. 9, pp. 1544-1555, Sept. 2014

[29] L. Wang and Z. Wang, "Mechanoreception for soft robots via intuitive body cues," Soft Robot., vol. 7, no. 2, pp. 198-217, Apr. 2020.

[30] F. Zhu, et al., "Failure handling of robotic pick and place tasks with multimodal cues under partial object occlusion," Front. Neurorobot., vol. 15 , p. 570507 , Mar. 2021

[31] F. Pedregosa et al., "Scikit-learn: machine learning in Python," J. Mach. Learn. Res., vol. 12, pp. 2825-2830, Nov. 2011. 